[0212-7199 (2003) 20: 11; pp 558-562] Copyright $\odot 2003$ ARAN EDICIONES, S.L.

An. MEd INTERNa (Madrid) Vol. 20, N. ${ }^{\circ} 11$, pp. 558-562, 2003

\title{
Neumonía en el anciano institucionalizado: criterios de derivación y/o clasificación pronóstica
}

\author{
A. B. BONILLA RODRÍGUEZ, M. J. GÓMEZ RODRÍGUEZ, F. ROBLES AGUDO, \\ E. VILALTA CASTEL, C. PEDREIRA COPÍN \\ Servicio de Medicina Familiar y Comunitaria. Área V. Servicios de Geriatría y de \\ Medicina Interna V. Hospital Gregorio Marañón H.G.U.G.M./Cantoblanco. Madrid. \\ Residencia PPMM. Colmenar Viejo. Madrid
}

PNEUMONIA IN ELDER INSTITUTIONALIZED PATIENTS: DERIVATION AND/OR PROGNOSTIC CLASSIFICATION CRITERION

\section{RESUMEN}

Objetivo: Conocer el perfil clínico de los pacientes con neumonía procedentes de Residencia comparándolo con los de neumonía adquirida en la comunidad (NAC) no institucionalizados e identificar los principales índices pronósticos de mortalidad.

Material y métodos: Estudio prospectivo longitudinal de todos los ancianos ingresados en el Hospital de Cantoblanco de Madrid durante el año 2001 diagnosticados de neumonía y clasificados según el índice pronóstico de Fine y los criterios de la SEPAR.

Resultados: De los 78 pacientes con neumonía, 27 procedían de Residencia, con una edad media de 86,85 $( \pm 6,43)$ años, frente a 83,11 $( \pm 5,87)$ años en los procedentes de domicilio $(p<0,05)$. De ellos, el $33,3 \%$ pertenecían a la clase IV y el $66,7 \%$ a la clase V de Fine. De todas las variables estudiadas, sólo la edad $(\mathrm{p}=0,03)$ y la hipoxemia $(\mathrm{p}=0,01)$ fueron estadísticamente significativas.

Conclusiones: Los pacientes con neumonía procedentes de Residencia tienen mayor edad y mayor prevalencia de morbi-mortalidad que los procedentes de su domicilio. En nuestro estudio, la edad y la hipoxemia son los dos factores pronósticos independientes asociados a mayor mortalidad.

PALABRAS CLAVE: Neumonía. Edad. Pronóstico. Mortalidad.

\section{ABSTRACT}

Objective: To know the clinical features of nursing home residents with pneumonia comparing with patients with Community-acquired pneumonia and identify the main prognostics index of mortality.

Material and methods: Longitudinal prospective study including all the elderly patients hospitalized in Cantoblanco Hospital of Madrid during the year 2001 for pneumonia and classified according to the Fine prognosis index and the SEPAR criteria.

Results: Of the 78 patients with pneumonia, 27 came from Residence, with an average of age of $86,85( \pm 6,43)$ years old, opposite to 83,11 $( \pm 5,87)$ years in patients with Community acquired pneumonia ( $p<0,05)$. Of all of them, 33,3\% belonged to class IV and $66,7 \%$ to class $V$ of Fine. Of all the variables studied, only the age $(p=0,03)$ and the hipoxemia $(p=0,03)$ were statistical significant.

Conclusions: Nursing home residents with pneumonia are older and have more prevalence of morbi-mortality than those with Community acquired pneumonia. In our study, the age and the hipoxemia were the two independent prognosis factors associate to more mortality.

Bonilla Rodríguez AB, Gómez Rodríguez, MJ, Robles Agudo F, Vilalta Castel E, Pedreira Copín C. Neumonía en el anciano institucionalizado: criterios de derivación y/o clasificación pronóstica. An Med Interna (Madrid) 2003; 20: 558-562.

\section{INTRODUCCIÓN}

La neumonía es la primera causa de muerte de etiología infecciosa en mayores de 65 años, ocupando el quinto lugar entre las causas de mortalidad global y el cuarto entre los motivos de hospitalización en mayores de 65 años.

La incidencia de la neumonía adquirida en la comunidad (NAC) es de tres a cinco veces superior en los individuos mayores de 65 años que en el resto de la población adulta. La incidencia de la neumonía en el medio residencial es similar a la hospitalaria, situándose en torno a 50 por cada 1.000 pacientes/año, siendo la segunda infección más frecuente en este medio.
Anualmente se atienden en nuestros hospitales de agudos unos 60.000 casos de NAC con una clara "geriatrización" (más del $50 \%$ en sujetos $\backslash 65$ años). La presentación de la enfermedad en la población anciana puede ser atípica, favoreciendo el retraso en el diagnóstico. Los síntomas típicos; fiebre, tos y dolor torácico pueden estar ausentes, siendo en ocasiones el cuadro confusional, el malestar inespecífico o el deterioro del estado general los únicos síntomas presentes, lo que conlleva muchas veces un retraso en el diagnóstico y en la instauración de un tratamiento eficaz. En el anciano, también son más frecuentes la presencia de bacteriemia, la implicación de microorganismos poco comunes en personas más jóvenes

Trabajo aceptado: 7 de mayo de 2003

Correspondencia: Ana Belén Bonilla Rodríguez. Ctra. de Canillas, 78, $3^{\circ}$ A. 28043 Madrid. 
con buen estado de salud previo, la aparición de complicaciones y el desenlace fatal. $(1,2)$

Los patógenos más frecuentemente encontrados en pacientes ancianos con NAC han sido: Streptococcus pneumoniae, Haemophilus influenzae, Clamydia pneumoniae, bacilos gramnegativos, Staphylococcus aureus y virus. Por el contrario, los más frecuentes en pacientes con neumonías adquiridas en residencia han sido los siguientes: flora aspirativa orofaríngea, S. pneumoniae, H.influenzae, S. aureus y bacilos gramnegativos. No obstante, se suelen observar notables diferencias etiológicas entre las series publicadas. En ello intervienen factores geográficos y estacionales, además de la edad del enfermo y su gravedad.

El tratamiento de la NAC es inicialmente empírico, modificado posteriormente en caso de mala evolución clínica o si los estudios microbiológicos lo desaconsejaran.

La severidad de las neumonías ha sido definida en numerosos estudios, tratándose de identificar una serie de factores asociados a una evolución con más complicaciones y a un aumento de la mortalidad. Entre ellos destaca el de García Ordóñez y cols., que destaca la importancia de la bacteriemia como factor pronóstico en la neumonía de la comunidad del anciano con una mortalidad mayor $(22,2$ vs $2,8 \%$; $p<0,001)$. (1) A menudo los médicos tienden a sobrestimar el riesgo de muerte de los pacientes con neumonía y eso se asocia con la decisión de hospitalizar a los pacientes de bajo riesgo.

A nivel residencial, la derivación al hospital se efectúa en función de la impresión de gravedad que el médico observe en el paciente y esto también puede conllevar excesivas tasas de hospitalización. Para evitar la subjetividad y ayudar al médico en la toma de decisiones, algunos autores han desarrollado distintos modelos de clasificación pronóstica: SEPAR (Sociedad Española de Neumología y Cirugía Torácica), Fine y cols., ATS (Sociedad Torácica Americana), IDSA (Sociedad de Enfermedades Infecciosas de América), etc. (3-6), en función de la edad del enfermo, la patología de base, los datos del examen físico y los resultados de laboratorio. En nuestro hospital de referencia se utiliza el índice pronóstico de Fine para identificar a los pacientes con neumonía adquirida en la comunidad que tienen alto riesgo de muerte y por lo tanto, teóricamente se beneficiarían de ingreso hospitalario.

El objetivo principal de nuestro trabajo es conocer el perfil clínico de los pacientes con neumonía, procedentes de Residencias, comparándolo con los pacientes ancianos con NAC no institucionalizados; así como, identificar, en este grupo de edad, los índices pronósticos más relevantes, asociados a una mayor mortalidad.

\section{MATERIAL Y MÉTODOS}

Se trata de un estudio prospectivo longitudinal, de un año de duración, realizado en un hospital de apoyo. Hemos incluido a todos los pacientes $\geq 75$ años que han ingresado en nuestro centro durante el año 2001 con el diagnóstico de neumonía adquirida en la comunidad y en particular, los procedentes de residencia. En todos los pacientes se recogieron de forma prospectiva y protocolizada datos personales, factores predisponentes para el desarrollo de neumonía (EPOC, hepatopatía, insuficiencia cardiaca, insuficiencia renal, ACV, demencia, diabetes, neoplasia, deterioro funcional, UPP, SNG), datos clínicos y de exploración física (fiebre, tos, expectoración, disnea, dolor pleurítico, alteración de la conciencia, aspiración, hipotensión, taquicardia y taquipnea), analíticos (leucocitos, hematocrito, glucemia, creatinina, sodio, proteínas, albúmina, PO2) y radiológicos que pudieran conllevar una evolución complicada de la NAC en el anciano.

A todos los pacientes con diagnóstico de NAC se les clasificó según el índice pronóstico de Fine (Tabla I) y los criterios de la SEPAR (Sociedad Española de Neumología y Cirugía Torácica) (6). En general, estas clasificaciones estratifican los episodios de NAC en 5 clases, de menor a mayor riesgo de mala evolución.

La estratificación del riesgo de muerte en 30 días o clasificación del riesgo según la puntuación de Fine es la siguiente:

\begin{tabular}{lcc} 
Clase & Puntuación total & $\begin{array}{c}\text { Medida a } \\
\text { considerar }\end{array}$ \\
\hline I & 0-60 puntos & Atención ambulatoria \\
II & 61-70 puntos & Atención ambulatoria \\
III & $71-90$ puntos & Atención hospitalaria \\
IV & $91-130$ puntos & Atención hospitalaria \\
V & $>130$ puntos & Atención hospitalaria \\
\hline
\end{tabular}

Fine MJ y cols. (3)

TABLA I

\section{SISTEMA DE PUNTUACIÓN DEL MODELO DE PREDICCIÓN QUE PERMITE ASIGNAR EL ENFERMO A UNA CLASE DE RIESGO I-V*}

\begin{tabular}{|c|c|}
\hline Característica & Puntuación asignada \\
\hline \multicolumn{2}{|l|}{ Factor demográfico } \\
\hline \multicolumn{2}{|l|}{ Edad } \\
\hline Hombre & Edad (años) \\
\hline Mujer & Edad (años) - 10 \\
\hline Residente en un hogar de ancianos & +10 \\
\hline \multicolumn{2}{|l|}{ Enfermedades coexistentes: } \\
\hline Enfermedad neoplásica & +30 \\
\hline Enfermedad hepática & +20 \\
\hline Insuficiencia cardíaca congestiva & +10 \\
\hline Enfermedad cerebrovascular & +10 \\
\hline Enfermedad renal & +10 \\
\hline \multicolumn{2}{|l|}{ Hallazgos del examen físico: } \\
\hline Estado mental alterado & +20 \\
\hline Frecuencia respiratoria $\backslash 30$ rpm & +20 \\
\hline Presión arterial sistólica < 90 mmHg & +20 \\
\hline Temperatura $<350 \backslash 40$ ËC & +15 \\
\hline Pulso \125 min & +10 \\
\hline \multicolumn{2}{|l|}{ Hallazgos de laboratorio: } \\
\hline $\mathrm{PH}$ arterial $<7,35$ & +30 \\
\hline Nitrógeno ureico $\backslash 30$ mg/dl & +20 \\
\hline Sodio $<130 \mathrm{mmol} / \mathrm{l}$ & +20 \\
\hline Glucosa $\backslash 250 \mathrm{mg} / \mathrm{dl}$ & +10 \\
\hline Hematocrito $<30 \%$ & +10 \\
\hline Presión parcial de oxígeno $<60$ mmHg & +10 \\
\hline Derrame pleural & +10 \\
\hline
\end{tabular}

Fine $M J$ y cols. (3)

* Para obtener la puntuación de un determinado paciente se suma su edad en años (la edad menos 10 en el caso de las mujeres) a los puntos de cada característica aplicable. 


\section{ANÁLISIS ESTADÍSTICO}

Para el análisis estadístico se ha realizado una descripción de las variables cualitativas mediante frecuencias y de las cuantitativas mediante media y desviación estándar. Se han comparado las variables cualitativas dos a dos mediante el test de la chi cuadrado o el test de Fischer cuando fue necesario. Las variables cuantitativas se han comparado mediante un $\mathrm{t}$ test o un análisis de la varianza cuando ha sido preciso.

\section{RESULTADOS}

De los 78 ingresos por neumonía, 27 (10 hombres y 17 mujeres) procedían de Residencia, con una edad media $( \pm \mathrm{DE})$ de 86,85 $( \pm 6,43)$ años, frente a 83,11 $( \pm 5,87)$ años en los procedentes de domicilio $(\mathrm{p}<0,05)$. Las patologías de base más frecuentes en los procedentes de Residencia, con diferencia significativa respecto a los demás, fueron: demencia: $70,4 \%$ $(\mathrm{p}<0,014)$, deterioro funcional: 85,2\% ( $\mathrm{p}<0,012)$, úlceras por presión: $44,4 \% \quad(\mathrm{p}<0,020)$ y sonda nasogástrica: $44,4 \%$ $(\mathrm{p}<0,020)$ (Fig. 1).

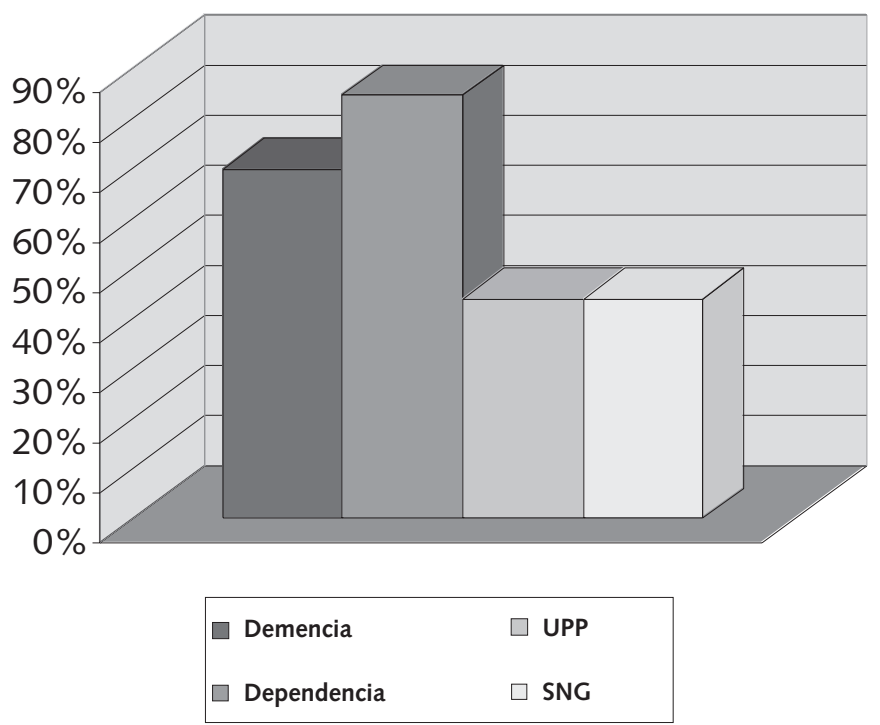

Fig. 1. Comorbilidad más frecuente en pacientes con neumonía procedentes de Residencias.

Los síntomas de presentación más frecuentes fueron: confusión $(70,4 \%)$, fiebre $(51,9 \%)$ y disnea $(55,6 \%)$. El $46,7 \%$ tenía una frecuencia respiratoria $\backslash 30$, el $65,4 \%$ insuficiencia respiratoria parcial y el $35 \%$ albúmina $\mathrm{f} 3$. La estancia media ( \pm DE) fue de 14,66 $\pm 13,99$ días y en los supervivientes de $16,42 \pm 16$. La mortalidad fue del $29,6 \%$ frente al $21,6 \%$ en los procedentes de domicilio, siendo la mortalidad global de $24,1 \%$.

Utilizando el índice pronóstico de Fine el 33,3\% pertenecían a la clase IV y el $66,7 \%$ a la clase V. A mayor categoría de riesgo en este índice aumenta la edad y la mortalidad ( $\mathrm{p}<$ 0.001 ), que era de $44,4 \%$ entre los 36 sujetos incluidos en el grupo V. También, a mayor categoría de riesgo en la clasifica- ción de la SEPAR era mayor la mortalidad ( $<$ < 0,019) y así, entre los 13 sujetos incluidos en el grupo $\mathrm{V}$ fue de 53,8\%.

En la tabla II podemos ver la distribución por grupos de riesgo. El coeficiente de correlación intraclase del índice de Fine con la clasificación de la SEPAR fue de 0,6755 $(0,50$ 0,75 : regular $\mathrm{y}>0,75$ : buena).

\section{TABLA ॥}

DISTRIBUCIÓN POR GRUPOS DE RIESGO, SEGÚN EL ÍNDICE DE FINE Y LA CLASIFICACIÓN DE LA SEPAR

\begin{tabular}{ccccc} 
Categoría & Episodios & Edad & Estancia & Mortalidad \\
\hline FINE & & & & \\
III & $8(10,2 \%)$ & 77,87 & 15,92 & 0 \\
IV & $34(43,6 \%)$ & 83,94 & 10,00 & $3(8,8 \%)$ \\
V & $36(46,2 \%)$ & 86,3 & 15,00 & $16(44,4 \%)$ \\
SEPAR & & & & \\
III & $3(3,8 \%)$ & 76,33 & 15,53 & 0 \\
IV & $62(79,5 \%)$ & 85,01 & 13,60 & $12(19,4 \%)$ \\
V & $13(16,7 \%)$ & 83,38 & 9,05 & $7(53,8 \%)$ \\
\hline
\end{tabular}

En el análisis bivariante la mortalidad se asocia significativamente con: edad, demencia, deterioro funcional, úlceras por presión, SNG, aspiración, fiebre, alteración del nivel de conciencia, hipotensión (TAS $<90 \mathrm{mmHg}$ ), taquipnea $>30$, hipoalbuminemia, acidosis, hipoxemia $\left(\mathrm{PO}_{2}<60 \mathrm{mmHg}\right)$. En el análisis multivariante mediante regresión logística de todas las variables que inicialmente se asociaban con la mortalidad, sólo la edad $(\mathrm{p}=0,03)$ y la hipoxemia $(\mathrm{p}=0,01)$, y marginalmente la fiebre $(p=0,09)$ y la aspiración $(p=0,07)$ permanecen como significativas en el modelo:

Variable OR (odds ratio)

\begin{tabular}{lc} 
Edad & 1,2231 \\
Hipoxemia & 16,59 \\
\hline
\end{tabular}

Por cada año que aumenta la edad, los sujetos tienen 1.2 veces más probabilidad de morir y los hipoxémicos tienen 16 veces más probabilidad de desenlace fatal que los no hipoxémicos.

\section{DISCUSIÓN}

La neumonía en el anciano es un importante problema de salud pública. Su elevada incidencia, 30 casos por 1.000 habitantes y año (mayor en residencias) se debe fundamentalmente a las enfermedades concomitantes existentes en estos pacientes y a su mayor discapacidad (7).

En nuestro estudio la edad más elevada, la mayor prevalencia de demencia, deterioro funcional, úlceras por presión y sonda nasogástrica fueron los rasgos clínicos diferenciales más significativos en el subgrupo de enfermos procedentes de residencias, datos que coinciden con estudios previos en los 
que se afirma que los pacientes ancianos con NAC no institucionalizados son más jóvenes, con menor deterioro cognitivo, mejor estado funcional y menor prevalencia de enfermedad cerebrovascular que los pacientes con neumonía adquirida en residencias (8).

La mortalidad de la NAC en el anciano, en la mayoría de las series (4-11), varía entre el 20-30\%. En nuestra serie, la mortalidad hospitalaria en el grupo de ancianos institucionalizados respecto a los procedentes del domicilio fue más elevada: 29,6 vs 21,6\%, respectivamente. La identificación de factores pronósticos es de gran importancia para evitar impresiones subjetivas a la hora de valorar la gravedad y/o ingreso de un anciano con NAC. En nuestra serie, las dos variables asociadas a mortalidad que permanecieron independientes fueron la edad y la hipoxemia. En los distintos trabajos publicados se encuentran datos contradictorios respecto al significado de la edad como factor pronóstico. Si bien, algunos autores han encontrado la edad como factor de mal pronóstico $(3,11)$; para otros, la edad por sí sola, no fue un factor significativo relacionado con el pronóstico (6). En nuestro estudio, los hipoxémicos tenían un riesgo 16 veces superior de un desenlace fatal respecto a los no hipoxémicos, En este sentido, Clemente y cols. (6), describen que si bien la hipoxemia es un factor de mal pronóstico, la presencia de dolor torácico sería un factor protector, probablemente relacionado con el nivel de conciencia del paciente. Para este autor (6), los factores pronósticos de mortalidad independientes en la NAC del paciente anciano fueron: la cifra de creatinina sérica superior a $1,2 \mathrm{mg} / \mathrm{dl}$, la inmovilización, la hipoxemia y la presencia de neoplasia previa.

Nuestros pacientes, por su pertenencia a las clases IV y V de Fine serían claros candidatos a manejo hospitalario por su mayor riesgo de muerte, circunstancia que no compartimos plenamente. Estos modelos se centran en la mortalidad como forma fundamental de medir la evolución de pacientes de cualquier edad e incluye diferentes factores predictivos de mala evolución, como edad muy avanzada, alteración del nivel de conciencia o vivir en residencia, premiando el ingreso. Pero la mayoría de estos modelos, no están desarrollados en población anciana y/o institucionalizada, en quienes la valoración de su situación funcional previa y la repercusión de los procesos agudos sobre su calidad de vida, tiene tanta o más importancia que la cantidad de vida; por lo que a veces, pueden favorecer el ingreso de pacientes mayores institucionalizados sin aportar mayor eficacia ni eficiencia que la obtenida con una atención integral en el medio residencial (10). De hecho, numerosos autores coinciden en afirmar que la mayoría de residentes que presentan neumonía podrían ser tratados satisfactoriamente en la propia residencia $(8,12,13)$. En recientes estudios se ha demostrado que incluso los pacientes con neumonías más severas pueden ser tratados en la Residencia sin diferencias significativas en cuanto a las cifras de mortalidad a los 30 días comparándolos con otros pacientes de igual severidad tratados en el hospital $(12,13)$. Otros autores han observado que el tratamiento de la neumonía moderada (< 40 r.p.m. al inicio del estudio) en el medio residencial supuso una mejoría del estado funcional a los dos meses (medida en actividades de la vida diaria) con respecto al tratamiento en el hospital. Así, los pacientes con menor dependencia funcional antes de la neumonía obtuvieron el mayor beneficio con el tratamiento en la residencia (14). En muchos casos se podría evitar, sin necesidad de adoptar decisiones negligentes, el consumo simultáneo de dos recursos (cama en hospital y cama en residencia) con el consiguiente ahorro de costes y la mejor utilización de los medios disponibles. No obstante, las residencias son muy heterogéneas en cuanto a los recursos humanos y materiales disponibles. Muchas residencias no están capacitadas para proporcionar antibióticos o sueroterapia intravenosos, bien sea por la falta de personal entrenado o por problemas económicos y en varios de los estudios citados los residentes recibieron terapia parenteral. Por ello, sería necesario desarrollar indicadores a pie de cama sobre la severidad de la infección en este medio y el beneficio global de su posible derivación al hospital. Los índices comentados requieren una serie de pruebas diagnósticas (hemograma, bioquímica, gasometria arterial, etc.) que no están disponibles en la práctica diaria para el manejo de las neumonías que debutan en residencias, haciendo menos funcionales los modelos hasta ahora disponibles.

La Sociedad Torácica Americana (ATS) (4) y la Sociedad de Enfermedades Infecciosas de América (IDSA) (5) han publicado unas guías para el manejo de la NAC. Sin embargo, estas guías no especifican datos para la neumonía adquirida en residencias y por ello los médicos no pueden extrapolar el uso de estas guías a la población de residentes con neumonía. Naughton y col han desarrollado una guía para el tratamiento de las neumonías en residencia que se basa principalmente en la práctica médica habitual en la comunidad y cuya información fue obtenida por una revisión retrospectiva de las historias clínicas de pacientes de 11 residencias de Búfalo (área de Nueva York) (13).

Se hace necesario el diseño de nuevos estudios para el desarrollo de índices pronósticos más adecuados en este grupo de edad, que ponderen no sólo la mortalidad sino también la función, como parámetros de evolución en los pacientes residentes con NAC.

\section{CONCLUSIONES}

Los pacientes con neumonía procedentes de Residencia tienen mayor edad y mayor prevalencia de demencia, deterioro funcional, úlceras por presión y sonda nasogástrica.

La mortalidad hospitalaria en este grupo es elevada, del $29,6 \%$, sin poder afirmar que los resultados en términos de mortalidad, función, manejo de síndromes geriátricos o confortabilidad, hubieran sido peores por su permanencia en la residencia.

En nuestra serie, la edad y la hipoxemia son los dos factores pronósticos independientes asociados a mayor mortalidad.

Son necesarios nuevos estudios para desarrollar índices pronósticos de la NAC en el paciente anciano institucionalizado que ponderen no sólo la mortalidad sino también la función y la confortabilidad. 


\section{Bibliografía}

1. García Ordóñez MA, Álvarez Hurtado F, Cebrián Gallardo JJ, López González JJ, Franquelo Vega M, Martínez González J, Juárez Fernández C. Neumonía bacteriémica de origen comunitario en el anciano. An Med Interna (Madrid) 1999; 345-348.

2. González Quijada S, Galdós Barroso M, Riego Valledor A, Fernández Älvarez G, Galindo Vicente $M^{\mathrm{a}} \mathrm{C}$. Neumonía en el anciano: ¿es atípica su presentación clínica? An Med Interna (Madrid) 2001; 18: 124-126.

3. Fine MJ, Auble TE, Yealy DM et al. A prediction rule to identify lowrisk patients with community-acquired pneumonia. N Engl J Med 1997; 336: 243-250.

4. Fang GD, Fine M, Orloff $J$ et al. New and emerging etiologies for community-acquired pneumonia with implications for therapy: A prospective multicenter study of 359 cases. Medicine (Baltimore) 1990; 69: 307316.

5. Marrie TJ, Durant H, Yates L. Community-acquired pneumonia requiring hospitalisation: 5-year prospective study. Rev Infect Dis 1989; 11: 586-599.

6. Clemente MG, Budiño TG, Seco GA, Santiago M, Gutiérrez M y Romero P. Neumonía adquirida en la comunidad en el anciano. Factores pronósticos. Arch Bronconeumol 2002; 38: 67-71.

7. Auble TE, Yealy DM. A prognostic rule for elderly patients admited with community-acquired pneumonia. Am J Med 2000; 108: 428.
8. Meehan TP, Chua-Reyes JM, Tate J, Prestwood KM, Cinto JD, Petrillo MK and Metersky ML. Process of care performance, patient characteristics and outcomes in elderly pacients hospitalised with Communityacquired or Nursing Home-acquired Pneumonia. Chest 2000; 117: 1378-85.

9. Lim WS, Lewis S, Macfarlane JT. Severity prediction rules in community acquired pneumonia: a validation study. Thorax 2000; 55: 219-223.

10. Fine MJ, Smith MA, Carsan CA, Mutha SS, Sankey SS, Weiss-feld LA, et al. Prognosis and outcomes of patients with community-acquired pneumonia. A meta-analysis. JAMA 1996; 275: 134-41.

11. Farr BM, Sloman AJ, Fisch MJ. Predicting death in patients hospitalized for community-acquired pneumonia. Ann Intern Med 1991; 115 : 428-36.

12. Mylotte JM, Naughton B, Maszarovics Z. Validation and application of the pneumonia prognosis index to nursing home residents with pneumonia. J Am Geriatr Soc 1998; 46: 1538-154.

13. Naughton BJ, Mylotte JM. Treatment guideline for nursing homeacquired pneumonia based on community practice. J Am Geriatr Soc 2000; 48: 82-88.

14. Fried TR, Gillick MR, Lipsitz LA. Short-term functional outcomes of long-term care residents with and without hospital transfer. J Am Geriatr Soc 1997; 45: 302-306. 